\title{
ANÁLISIS DEL IMPACTO DE LA COLABORACIÓN INTERNACIONAL EN EL ÁREA DE ESTUDIOS EMPRESARIALES
}

\section{ANALYSIS OF THE IMPACT OF INTERNATIONAL COLLABORATION IN BUSINESS STUDIES AREA}

Antonio Velez-Estevez (Universidad de Cádiz) ${ }^{1}$

Pablo Garcia-Sanchez (Universidad de Granada) ${ }^{2}$

\section{Resumen:}

Con el auge de las nuevas tecnologías y medios de colaboración, nos estamos adentrando en una nueva etapa en el ámbito científico en el que los académicos buscan aprovechar las ventajas que la colaboración internacional ofrece. Una de esas ventajas es el aumento del impacto, medido en forma del número de citas, de aquellos trabajos realizados en conjunto por más de un país. En este trabajo se pretende demostrar que también ocurre en el ámbito de los estudios empresariales realizado en España. Para ello, se ha medido el número medio de citas y el índice h de todos los artículos publicados en este campo por autores españoles en distintos niveles de colaboración: local (los autores pertenecen a la misma afiliación), nacional (los autores pertenecen a más de una afiliación, todas de España) e internacional (al menos uno de los autores es de un país extranjero). Los resultados demuestran que el mayor número medio de citas y el mayor índice h se obtiene en el nivel de colaboración internacional. Además, se ha realizado una comparación de las distintas universidades españolas, mostrando que existen diferencias entre ellas respecto al grado de colaboración internacional. Este estudio pretende ser la base para que los agentes interesados en mejorar las políticas científicas de este campo en España obtengan una visión global del estado de colaboración internacional y puedan profundizar en el futuro para mejorar sus políticas.

Palabras clave: Colaboración internacional, colaboración nacional, bibliometría, cienciometría, índice h.

Códigos JEL: O32.

\begin{abstract}
:
\footnotetext{
1 antonio.velezestevez@alum.uca.es, Universidad de Cádiz.

2 pablogarcia@ugr.es, Universidad de Granada.

Recibido 8 de abril de 2020. Aceptado 8 de junio de 2020.
}

With the rise of new technologies and means of collaboration, we are entering a new phase in the scientific field in which academics are seeking to take advantage of the benefits that international collaboration offers. One such advantage is the increased impact, measured in the number of citations, of those works carried out jointly by more than one country. This paper aims to demonstrate that this is also the case in the field of business studies carried out in Spain. To this end, we have measured the average number of citations and the h-index of all articles published in this field by Spanish authors at different levels of collaboration: local (the authors belong to the same affiliation), national (the authors belong to more than one affiliation, all from Spain) and international (at least one of the authors is from a foreign country). The results show that the highest average number of citations and the highest h-index is obtained at the international collaboration 
level. In addition, a comparison of the different Spanish universities was made, showing that there are differences between them with respect to the degree of international collaboration. This study is intended to be the basis for agents interested in improving scientific policies in this field in Spain to obtain a global vision of the state of international collaboration and to be able to go further into the future to improve their policies.

Keywords: International collaboration, national collaboration, bibliometrics, scientometrics, h-index.

JEL Codes: O32.

\section{INTRODUCCIÓN}

Se suele pensar que la investigación se suele realizar de forma doméstica, es decir, que los investigadores de una única universidad son los firmantes de cada artículo científico. Sin embargo, con el auge de las nuevas tecnologías que facilitan la colaboración y la compartición de resultados entre investigadores, independientemente de su lugar de trabajo, estamos asistiendo a la llamada cuarta etapa de la investigación, donde prima la colaboración internacional (Fortunato et al., 2018).

No solo aquellos científicos que han cambiado de afiliación internacional a lo largo de su carrera obtienen más impacto que aquellos que se quedan en un único país (Sugimoto et al., 2017), sino que, además, se ha probado que los artículos desarrollados por miembros de más de un país obtienen más citas que los artículos escritos por equipos de un único país (Persson et al., 2004; García-Sánchez y Cobo, 2018).

Es por ello que distintos agentes, como gobiernos o agencias de evaluación, están ajustando sus políticas para favorecer la colaboración internacional, por lo que se hacen necesarios nuevos estudios para que estos agentes puedan tomar las decisiones adecuadas (Castillo y Powell, 2018).

Principalmente es necesario estudiar el impacto internacional en distintas áreas, como los distintos campos de investigación, países o comunidades de colaboración. De hecho, como se muestra en (Polyakov et al., 2017; Wagner et al., 2017) existen diferencias respecto al campo de investigación: algunos campos tienen un porcentaje de colaboración internacional (artículos firmados por autores de más de un país) completamente diferente al de otros campos. Es por ello que el estudio de cada campo por separado puede permitir obtener conocimiento sobre la estructura, redes de colaboración e impacto de su investigación.

Esto nos ha motivado a realizar un estudio de los distintos perfiles de colaboración del campo de estudios empresariales en España. Estos artículos pueden clasificarse en los siguientes perfiles según la afiliación de sus autores: local (los firmantes son de una única universidad), nacional (los firmantes son de varias universidades de España) e internacional (al menos hay una universidad no española en la lista de firmantes).

El objetivo de este artículo es demostrar que el impacto, medido como el número de citas, de los artículos en el campo de estudios empresariales en España, es mayor cuando la colaboración es internacional.

Para demostrarlo se realizará un análisis descriptivo para los tres perfiles mencionados, así como la evolución de la colaboración en el tiempo de las instituciones universitarias españolas. Para ello se utilizarán todos los artículos del campo de estudios empresariales de la base de datos Web of Science (WoS). No obstante, es necesario indicar 
que aunque la internacionalización puede ser una característica determinante a la hora de medir el impacto, siempre será necesario tener en consideración otros factores como, por ejemplo, la calidad de los resultados, accesibilidad, lenguaje o impacto de las revistas entre otros muchos (Tahamtan et al., 2016).

El resto del artículo se estructura como sigue. Después del estado del arte en el ámbito de la colaboración científica internacional, se describe la metodología utilizada, explicando cómo se han obtenido los datos a analizar. A continuación, se describe el conjunto de datos utilizado y se analizan y discuten los resultados. Finalmente, se presentan las conclusiones y futuras líneas de trabajo.

\section{ESTADO DEL ARTE}

Debido al incremento de la producción científica, y su disponibilidad en distintas bases de datos en línea, así como la movilidad de los científicos y el aumento de financiación, han producido que surjan nuevas oportunidades de cara a la evaluación de la ciencia (Milojević, 2015). El uso de distintas técnicas y herramientas para obtener información de esta gran cantidad de datos puede ayudar a los distintos agentes a comprender las relaciones entre los científicos, instituciones e ideas (Fortunato et al., 2018). Como se ha indicado en la introducción, la cuarta etapa de la investigación está motivada por la colaboración $\mathrm{y}$, por lo tanto, los investigadores cuentan con investigadores de otros países para avanzar en sus carreras y campos de investigación (Moed et al., 2013).

Existen distintos trabajos que han utilizado la cienciometría para explicar las tendencias y características para estudiar las colaboraciones y el impacto (Tahamtan et al., 2016; Asubiaro, 2019). Por ejemplo, se ha demostrado que artículos desarrollados por grupos internacionales reciben más citas (Persson et a.l, 2004). Concretamente, un trabajo muy similar al presentado aquí fue desarrollado por García-Sánchez y Cobo (2018). En ese artículo se utilizó la base de datos Dimensions.ai para obtener todos los artículos realizados por miembros de las universidades andaluzas en el periodo 2010-2018, y se compararon los distintos niveles de colaboración (local, nacional, e internacional). Los autores confirmaron que, al aumentar el nivel de colaboración, aumenta el número medio de citas. Sin embargo, el estudio sólo se centró en un pequeño grupo de universidades, y se aplicó a todos los campos científicos.

Además, no sólo el número de países afecta al impacto, sino que el nivel económico de los países también es un factor que afecta al número de citas (Ni y An, 2018), así como la edad de las universidades participantes (Khor y Yu, 2016).

De hecho, se ha mostrado que existen diferencias en el impacto internacional entre diferentes áreas. En Polyakov et al. (2017) se tuvo en cuenta el tiempo de publicación de los artículos, junto con análisis semántico para distinguir distintos tópicos y se demostró que el efecto de los distintos tipos de colaboración también varía dependiendo del tópico dentro un mismo campo.

Otros autores, han realizado estudios similares al presentado en el ámbito de las ciencias sociales (Sooryamoorthy, 2017) (concretamente en el área geográfica de África), la salud ocupacional (Ni y An, 2018) o la bioquímica (Sud y Thelwall, 2015), mostrando resultados similares a los de este trabajo.

En el caso concreto de los estudios empresariales, en Fang et al. (2020) se presenta un estudio muy completo sobre las escuelas de negocios chinas, a partir de sus artículos publicados en un grupo de revistas. También se demostró el impacto positivo del número de países que colaboran con la institución. 
A la vista de los anteriores trabajos, y tomando ideas de ellos, en este trabajo está centrado en el campo de estudios empresariales en España, para obtener una visión más precisa de este campo y área geográfica, para que las entidades interesadas puedan estudiar las oportunidades que la internacionalización puede ofrecer.

\section{METODOLOGÍA}

Para conseguir el propósito de este artículo se han dividido los datos descargados en tres niveles de colaboración distintos:

- Local. Artículos en los que solo colabora una institución española.

- Nacional. Artículos en los que colaboran distintas instituciones españolas.

- Internacional. Artículos en los que colabora más de un país, es decir, España más cualquier otro.

Para medir el impacto académico que tienen los niveles de colaboración anteriormente mencionados en el campo de estudios empresariales, se estudiarán las citas de los artículos publicados por instituciones universitarias españolas en dicha categoría para la base de datos Web of Science.

Con este objetivo, se ha usado usado la API de la Web of Science que permite descargar los artículos realizando peticiones vía REST desde cualquier lenguaje de programación. Para ello se ha diseñado la siguiente consulta:

\section{"WC="Business" AND CU="SPAIN" AND DT=(Article OR Review)"}

Esta consulta devuelve un conjunto de artículos en los que se incluyen datos como el identificador (UID), las afiliaciones de los autores, el año, así como las veces que se ha citado el artículo y algunos datos más.

Estos datos se tienen que procesar con el objetivo de que cumplan lo que se esperaba de la consulta, es decir, que al menos cada artículo contenga a un país y que también contenga al menos a España, y además deben eliminarse aquellas entradas con valores nulos, así como los documentos duplicados. Se han filtrado también los documentos que tenían el valor de los países y de las instituciones vacío. Por otra parte, se han normalizado todos los nombres de los países y organizaciones a mayúsculas y se han sustituido Wales, Scotland, North Ireland y England por United Kingdom.

Además, se han filtrado los artículos dejando aquellos que hayan sido firmados por alguna institución española cuyo nombre contenga UNIV. De esta forma se puede asegurar que el conjunto de datos cumple con el estudio de instituciones universitarias españolas.

Por otra parte, para cada uno de los artículos en el conjunto de datos se ha normalizado el número de citas respecto de la media de citas de todos los artículos publicados en el mismo año obteniendo finalmente el Mean Normalised Citation Score (MCNS). Cuando el valor del MCNS de un artículo está por encima de la unidad indica que el artículo tiene un impacto por encima de la media de las citas de los artículos de ese mismo año.

En cuanto al estudio global del conjunto de datos se ha realizado lo siguiente:

- Un análisis descriptivo del conjunto de datos descargado.

- Evolución del número de artículos por año.

- Evolución de países e instituciones colaboradoras por año.

- Top 15 de países colaboradores con instituciones universitarias españolas. 
- Top 15 de instituciones colaboradoras con instituciones universitarias españolas.

- $\quad$ Evolución temporal del impacto según el nivel de colaboración a partir del MCNS.

Además, se han calculado por cada institución universitaria española distintos indicadores que pretenden servir de base para demostrar el impacto de cada uno de los niveles de colaboración que nos permitan comparar los mismos. Estos indicadores son los siguientes:

- $\quad$ La distribución de la colaboración.

- La media de citas de sus artículos por nivel de colaboración.

- $\quad$ El índice h por nivel de colaboración.

Los datos obtenidos en JSON han sido procesados, analizados y convertidos con la biblioteca Pandas de Python.

\section{RESULTADOS Y DISCUSIÓN}

La consulta se realizó el día 28 de febrero de 2020, y se obtuvieron 11.664 artículos. Tras el procesamiento indicado en la sección anterior resulta en un total de 10.714 artículos.

Los resultados muestran claras diferencias en las citas por cada nivel de colaboración tal y como se puede comprobar en la tabla 1 . Inicialmente se puede observar que en el campo de estudios empresariales prima la colaboración local, con cerca de la mitad de artículos (48 por cien) firmados por autores de una única institución. Sin embargo, el siguiente nivel más común es el de la colaboración internacional, con el 36 por cien de artículos. La media de países que colaboran en el nivel internacional es de 2,53 mientras que el número de instituciones es ligeramente superior a 3 .

TABLA 1 RESUMEN DEL ANÁLISIS DESCRIPTIVO DEL CONJUNTO DE DATOS PARA LOS TRES NIVELES DE COLABORACIÓN.

\begin{tabular}{|l|l|l|l|l|}
\hline & LOCAL & NACIONAL & INTERNACIONAL & GLOBAL \\
\hline Número de artículos & 5178 & 1716 & 3820 & 10714 \\
\hline Porcentaje de artículos & $48 \%$ & $16 \%$ & $36 \%$ & $100 \%$ \\
\hline Índice H & 93 & 68 & 115 & 148 \\
\hline $\begin{array}{l}\text { Media de citas por } \\
\text { artículo }\end{array}$ & 11,73 & 12,98 & 19,64 & 14,75 \\
\hline $\begin{array}{l}\text { Media de instituciones } \\
\text { por artículo }\end{array}$ & - & 2,19 & 3,01 & 1,91 \\
\hline $\begin{array}{l}\text { Media de países por } \\
\text { artículo }\end{array}$ & - & - & 2,53 & 1,54 \\
\hline
\end{tabular}

Fuente: Elaboración propia.

Con respecto al impacto (número de citas e índice h) se demuestra que en nivel de colaboración internacional se consiguen los valores más altos en estos dos campos, prácticamente doblando el número de citas del nivel local. Con respecto al nivel nacional, el número de citas medio también es mayor que el nivel local. Sin embargo, este nivel obtiene el menor índice h de los tres niveles. Esto puede ser debido a que sólo el 16 por cien de artículos se publican a nivel nacional, respecto al 48 por cien del nivel local. 
Además, se puede observar cómo considerando todos los artículos el índice h es superior al de la colaboración internacional a pesar de tener una media de citas menor.

Con respecto a la evolución de la media de países colaboradores y media de instituciones colaboradoras, la figura 1 muestra como existe una clara ascendencia en ambos valores. Sin embargo, en los primeros años representados hay mucha variabilidad debido al menor número de artículos durante esos años, como puede verse en la figura 2, cuando el número de artículos rondaba las pocas unidades antes de 2004, siendo este año el inicio de un gran crecimiento.

\section{FIGURA 1 EVOLUCIÓN DE LA COLABORACIÓN RESPECTO A LA MEDIA DE NÚMERO DE PAÍSES E INSTITUCIONES POR AÑO.}

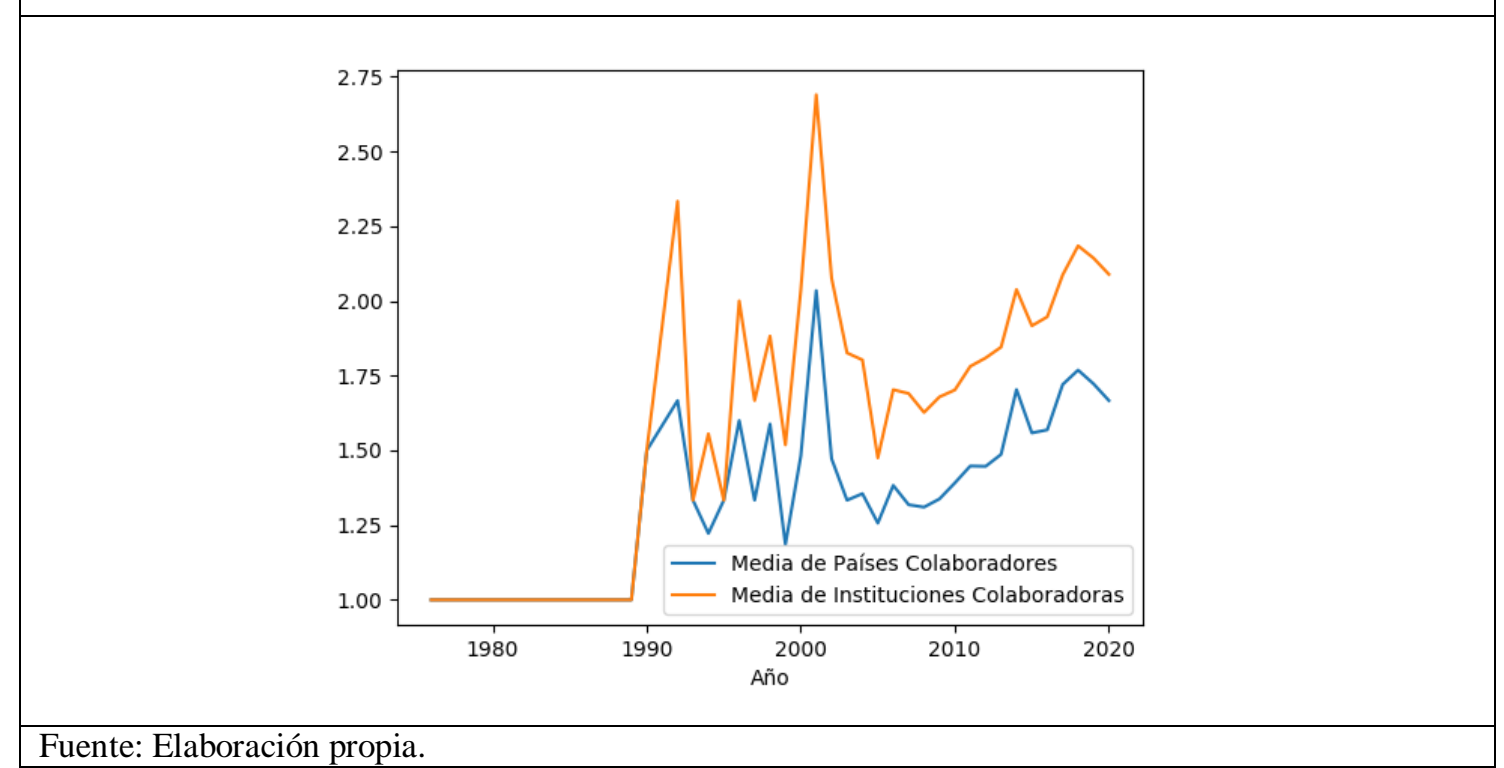

FIGURA 2 EVOLUCIÓN DEL NÚMERO DE ARTÍCULOS POR AÑO.

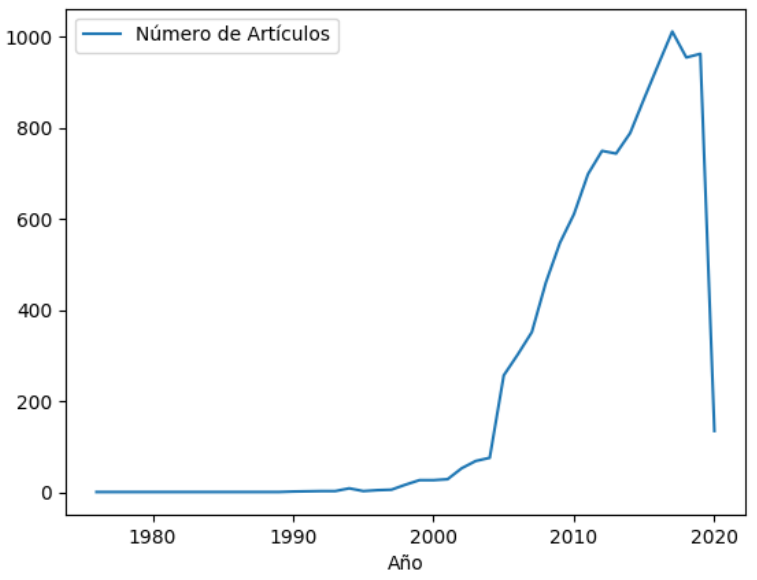

Fuente: Elaboración propia.

A continuación, se realizará un análisis descriptivo de las distintas métricas por instituciones de investigación y universitarias españolas. La figura 3 muestra que el porcentaje por institución es muy variado, habiendo 9 instituciones con más de la mitad 
de sus artículos realizados a nivel internacional, mientras que otras no tienen ninguno. La Universidad Pontificia de Salamanca es la que mayor porcentaje obtiene, un 71,43 por cien de sus artículos se han realizado con países extranjeros. Sin embargo, la gran mayoría de instituciones se mueven entre el 20 y el 40 por cien. La Universidad de Jaén, en concreto, cuenta con muy pocos artículos en el nivel internacional, con respecto al resto de instituciones.

\section{FIGURA 3 PORCENTAJE DE ARTÍCULOS EN CADA NIVEL DE COLABORACIÓN POR INSTITUCIONES UNIVERSITARIAS ESPAÑOLAS.}

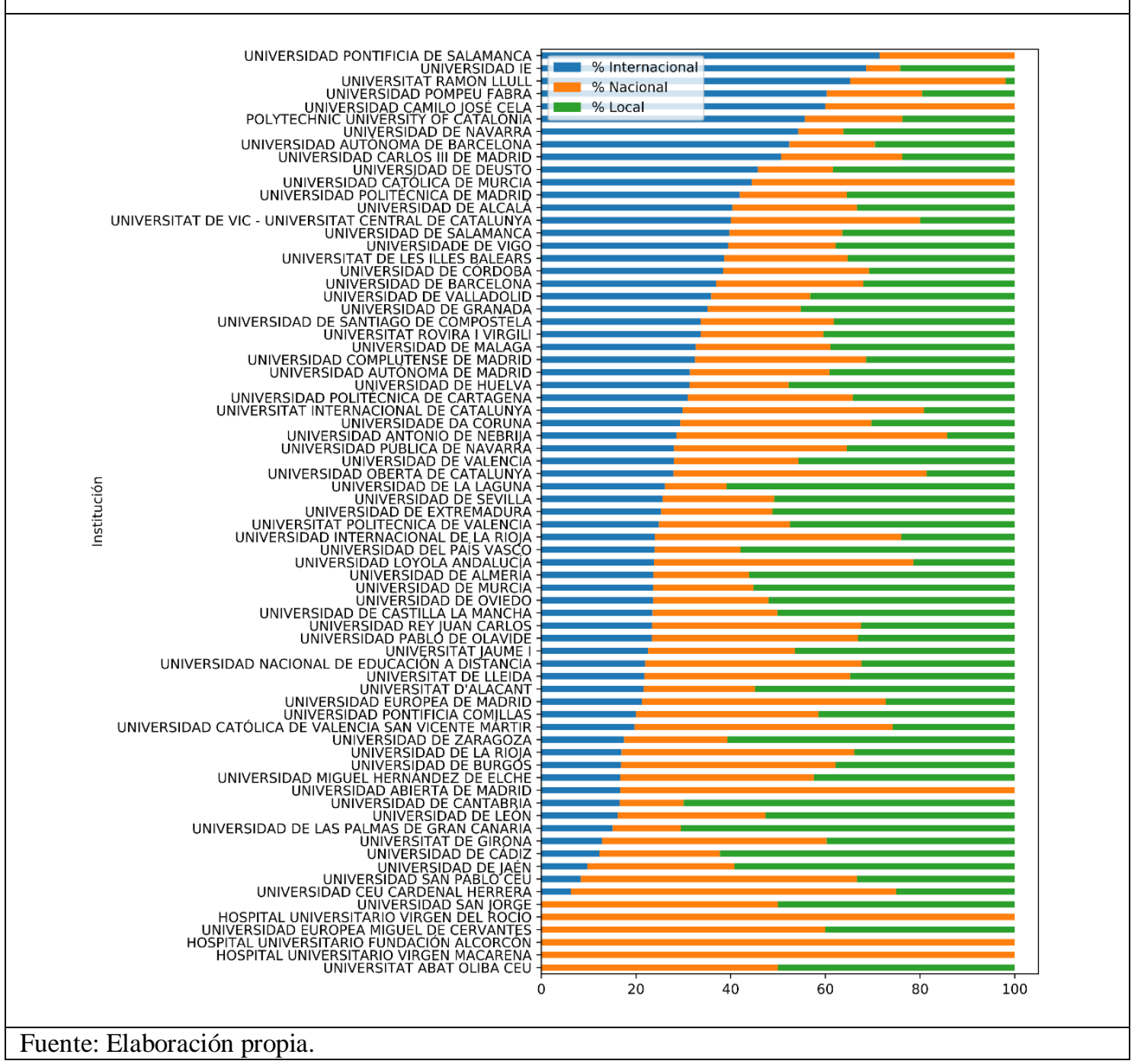

También existe variabilidad con respecto a los otros dos niveles: en algunas instituciones el nivel local es mayor que el internacional y viceversa, es decir, aunque en la tabla 1 el porcentaje de artículos en el nivel nacional era el menor en general, en aproximadamente un tercio de las afiliaciones esto no se cumple.

Con respecto al número medio de citas, la figura 4 muestra la media de cada nivel por cada afiliación. Como puede verse, el mayor número de citas corresponde al nivel internacional para más de un tercio de las instituciones, siendo los mayores valores, los correspondientes a este nivel. La Universidad de Jaén cuenta con una extraordinaria media de citas internacional, pero esto es debido a que cuenta con 10 artículos en este 
nivel, y uno de ellos tiene más de 1.000 citas. Es por ello que es necesario utilizar la métrica del índice h para obtener una visión menos sesgada de la importancia de cada nivel.

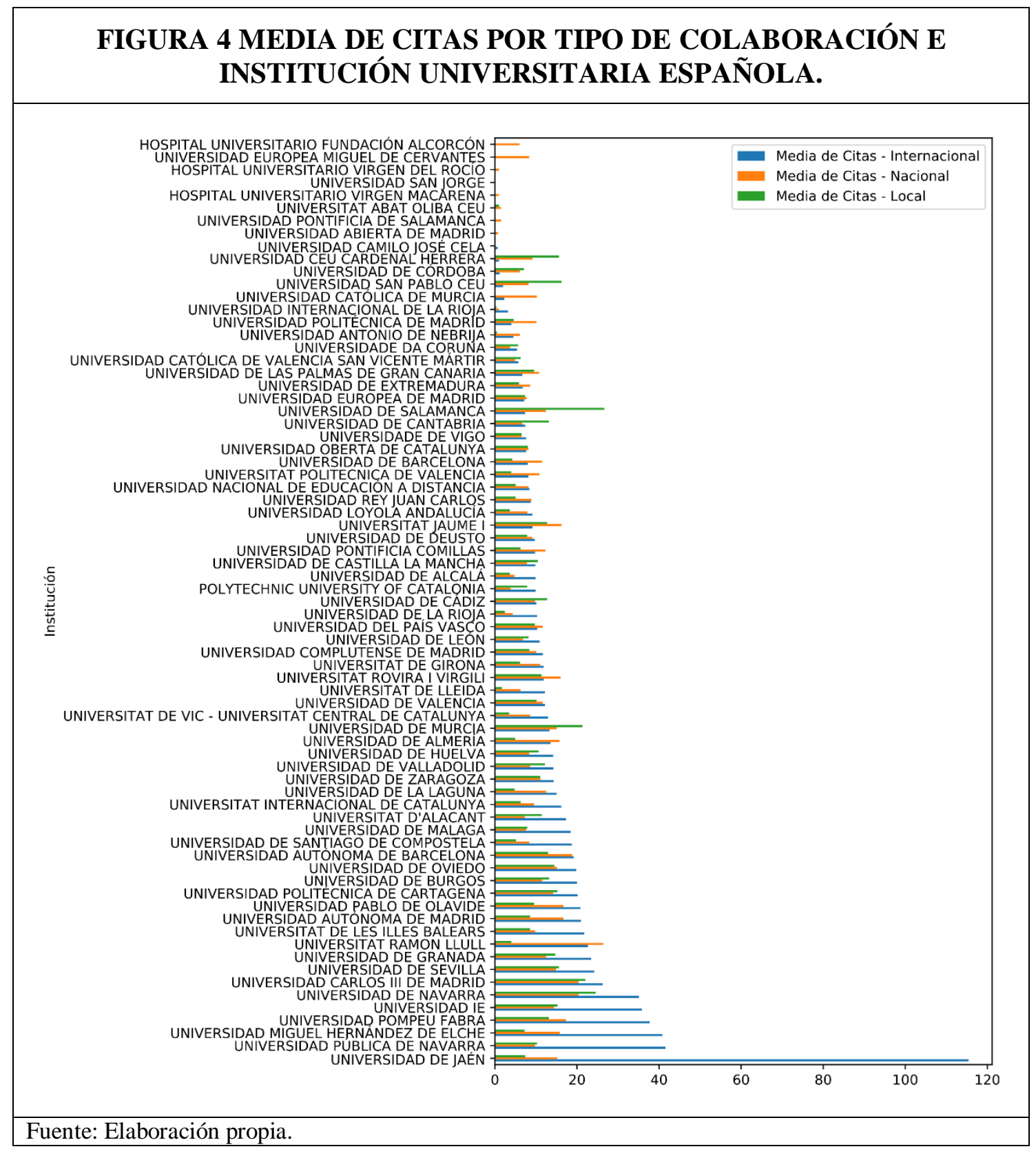

La figura 5 muestra el índice h de los artículos de cada nivel, ordenada por el índice $\mathrm{h}$ de dicho nivel. Aquí puede observarse como los mayores niveles de índice $\mathrm{h}$ corresponden al nivel internacional. Aunque algunas universidades mantienen su orden en los puestos más altos, otras, como la mencionada Jaén, o Elche, están en lugares más intermedios. También puede verse como algunas universidades tienen más índice h en el nivel local, algunas incluso compitiendo con el elevado índice h que obtienen aquellas en la parte baja de la Figura, como, por ejemplo, la Universidad de Murcia, con un índice h local de 35. 




Con respecto a los países que más colaboran con las instituciones españolas, la figura 6 muestra el número de artículos escritos en colaboración con otros países. Como puede verse, el país con el que más se colabora es claramente Estados Unidos de América. 7 de los 15 países pertenecen a la Unión Europea (que en su conjunto doblan a los escritos en colaboración con EE.UU.). Canadá es el primer país fuera de la UE y EE.UU. con el que más se colabora, existiendo también relación en el top 15 con otros países de Latinoamérica, como Colombia, Chile, México y Brasil. 


\section{FIGURA 6 TOP 15 PAÍSES COLABORADORES EN LOS ARTÍCULOS ESPAÑOLES POR NÚMERO DE ARTÍCULOS.}



Fuente: Elaboración propia.

En la figura 7 se muestra de forma ordenada las instituciones con las que más se colabora en esta área. A pesar de ser EE.UU. el país con mayor número de artículos, solamente 7 de las 15 instituciones mostradas en esta tabla corresponden a este país, por lo que la investigación está más distribuida en varios centros. En el caso de Reino Unido, un tercio de los artículos publicados se reparten entre 3 afiliaciones (dos de ellas en la ciudad de Londres). En países más pequeños, como Países Bajos e Italia, aproximadamente un tercio de las publicaciones en común se realizan en una única universidad (Erasmus University Rotterdam y Bocconi University, respectivamente).

FIGURA 7 TOP 15 INSTITUCIONES EXTRANJERAS COLABORADORAS EN LOS ARTÍCULOS ESPAÑOLES POR NÚMERO DE ARTÍCULOS.

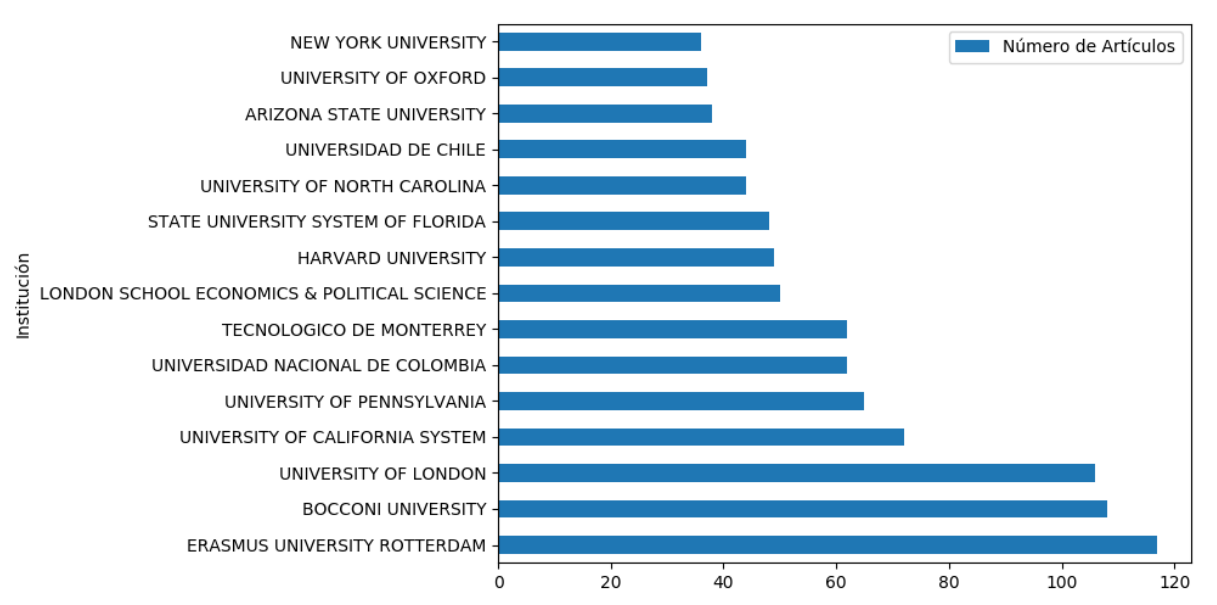

Fuente: Elaboración propia. 
Por último, se ha considerado un análisis temporal del impacto según los niveles de colaboración, como se puede comprobar en la figura 8. En esta figura se puede ver cómo el impacto de la colaboración internacional de forma global en España ha sido mayor que en los demás niveles de colaboración de forma sostenida desde el año 1999, excluyendo el año 2004. Además, ha estado por encima de la media global de citas del área, puesto que supera a uno en el periodo indicado anteriormente.

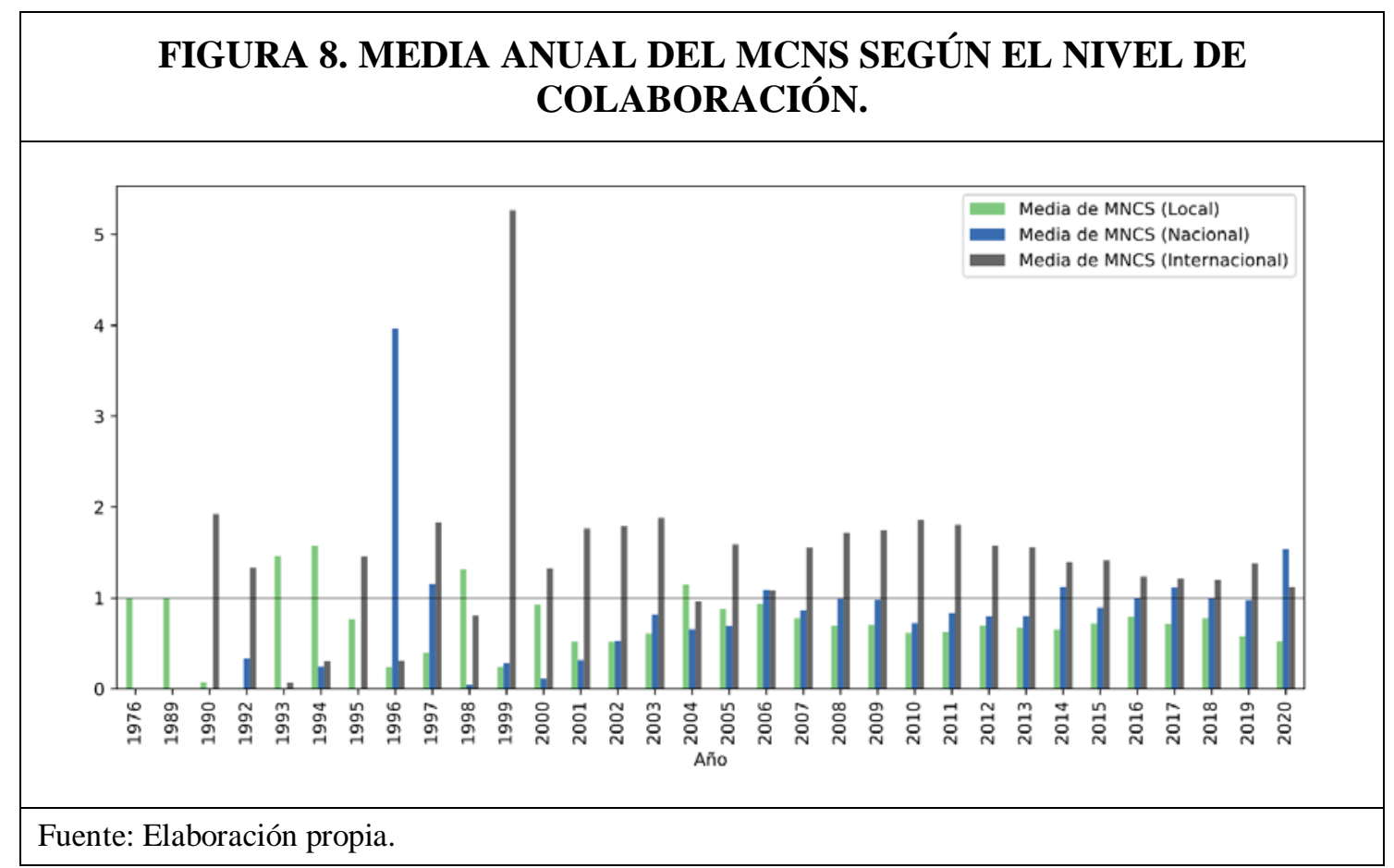

\section{CONCLUSIONES}

Gracias a las nuevas tecnologías, el mundo académico está virando hacia una nueva forma de realizar ciencia, en la que la colaboración científica internacional está adquiriendo más protagonismo. Aunque existen distintos estudios que han demostrado que la cooperación internacional aumenta el impacto de las publicaciones firmadas por miembros de distintos países, no existía ningún análisis en el campo de estudios empresariales en España.

Para demostrar que esto se cumple, se han descargado los artículos publicados por investigadores españoles pertenecientes al campo "Business” de la Web of Science.

A partir de ese conjunto de datos, se demuestra que los artículos firmados por miembros de distintos países, además de España, obtienen más citas que aquellos sólo firmados por miembros de instituciones españolas. Este último conjunto también obtiene más citas de media que los artículos publicados a nivel local (los autores pertenecen a una única institución).

También se ha comprobado como en este campo hay un incremento en el número de colaboración a nivel internacional con el tiempo, además de un importante crecimiento del número de artículos.

Además, se ha realizado un estudio de las distintas universidades españolas, mostrando una gran diferenciación entre ellas: mientras algunas publican mayormente artículos internacionales, otras lo hacen de forma local. Salvo excepciones, el mayor 
impacto se recibe en el nivel internacional por cada universidad, siendo aquellas universidades con más impacto aquellas con mayor porcentaje de artículos internacionales.

No obstante, y aunque hemos mostrado cómo la media del número de citas es mayor en el tipo de colaboración internacional no podemos confirmar que firmar trabajos por autores de distintos países sea la única fuente del impacto. Es necesario por lo tanto estudiar con más profundidad su relación con otros elementos, como fuentes de financiación, idioma o accesibilidad de los trabajos, así como la temática, interés y calidad de los resultados, entre otros muchos factores.

El impacto positivo sostenido en el tiempo de la colaboración internacional en el campo de estudios empresariales en las distintas instituciones universitarias españolas refleja una tendencia interesante. Es preciso indicar que iniciar políticas que fomenten este tipo de colaboración podría tener un impacto positivo en la visibilidad de la investigación de estas universidades.

Por otra parte, vistos los resultados, resulta innegable que incrementar las relaciones de investigación con las instituciones y países que se muestran en el top 15 de este estudio es de vital importancia. En este sentido mejorar las relaciones con Estados Unidos y Reino Unido, y concretamente con las instituciones de estos países que se muestran en nuestro estudio, podría incrementar el impacto de las investigaciones en este campo.

Este trabajo pretende ser la base de futuras mejoras, por ejemplo, realizar un estudio de qué países o conjuntos de países reciben más impacto o estudiar las relaciones de colaboración de estas universidades de una forma más profunda. También se aplicarán otras técnicas de bibliometría, como el análisis de redes de co-palabras, al igual que se realizó en (Velez-Estevez, García-Sánchez, Moral-Munoz y Cobo, 2020), para descubrir qué tópicos son los que obtienen más impacto en cada uno de los niveles. Otras características, como el nivel de financiación de las universidades, su tamaño, o su año de fundación, también pueden aportar información a los agentes interesados en mejorar el impacto en este campo.

\section{BIBLIOGRAFÍA}

Asubiaro, T. (2019). How collaboration type, publication place, funding and author's role affect citations received by publications from Africa: A bibliometric study of LIS research from 1996 to 2015. Scientometrics, 120(3), 1261-1287.

Castillo, J. A. y Powell, M. A. (2018). Research Impact and International Collaboration: A Study of Ecuadorian Science . Journal of Hispanic Higher Education.

Fang, W., Dai, S. y Tang, L. (2020). The impact of international research collaboration network evolution on Chinese business school research quality. Complexity.

Fortunato, S., Bergstrom, C. T., Börner, K., Evans, J. A., Helbing, D., Milojević, S., Barabási, A.-L. (2018). Science of Science. Science, no 359, pp. 637).

García-Sánchez, P. y Cobo, M. J. (2018). Measuring the Impact of the International Relationships of the Andalusian Universities Using Dimensions database. International Conference on Intelligent Data Engineering and Automated Learning, pp. 138-144.

Khor, K. A. y Yu, L.-G. (2016). Influence of international co-authorship on the research citation impact of young universities. Scientometrics, vol. 107, $\mathrm{n}^{\mathrm{o}} 3$, pp. 10951110.

Milojević, S. (2015). Quantifying the cognitive extent of science. Journal of Informetrics, vol. 9, no 4, pp. 962-973. 
Moed, H. F., Aisati, M. y Plume, A. (2013). A Studying scientific migration in Scopus. Scientometrics, vol. 94, $\mathrm{n}^{\circ}$ 3, pp. 929-942.

Ni, P. y An, X. (2018). Relationship between international collaboration papers and their citations from an economic perspective. Scientometrics, vol. 116, $\mathrm{n}^{\circ} 2$, pp. 863877.

Persson, O., Glänzel, W. y Danell, R. (2004). Inflationary bibliometric values: The role of scientific collaboration and the need for relative indicators in evaluative studies. Scientometrics, vol. 60, $\mathrm{n}^{\circ}$ 3, pp. 421-432.

Polyakov, M., Polyakov, S. y Iftekhar, M. S. (2017). Does academic collaboration equally benefit impact of research across topics? The case of agricultural, resource, environmental and ecological economics. Scientometrics, vol. 113, n ${ }^{\circ} 3$, pp. 13851405.

Sooryamoorthy, R. (2017). Do types of collaboration change citation? A scientometric analysis of social science publications in South Africa. Scientometrics, vol. 111, $\mathrm{n}^{\mathrm{o}} 1$, pp. 379-400.

Sud, P. y Thelwall, M. (2015). Not all international collaboration is beneficial: The Mendeley readership and citation impact of biochemical research collaboration. Journal Of The Association For Information Science And Technology, vol. 67, ${ }^{\circ}$ 8, pp. 1849-1857.

Sugimoto, C., Robinson-Garcia, N., Murray, D., Yegros-Yegros, A., Costas, R. y Larivière, V. (2017). Scientists have most impact when they're free to move. Nature, vol. 550, no 7674, pp. 29-31.

Tahamtan, I., Safipour Afshar, A. y Ahamdzadeh, K. (2016). Factors affecting number of citations: a comprehensive review of the literature. Scientometrics, vol. $107, \mathrm{n}^{\circ} 3$, pp. 1195-1225.

Velez-Estevez, A., García-Sánchez, P., Moral-Munoz, J. y Cobo, M. J. (2020). Thematical and Impact Differences Between National and International Collaboration on Artificial Intelligence Research. IEEE Conference on Evolving and Adaptive Intelligent Systems (EAIS2020).

Wagner, C. S., Whetsell, T. A. y Leydesdorff, L. (2017). Growth of international collaboration in science: revisiting six specialties. Scientometrics, $\mathrm{n}^{0} 110$, pp. 1633-1652. 